\title{
Searches for new heavy resonances in final states with leptons and photons at CMS
}

\author{
Benjamin Radburn-Smith* ${ }^{* \dagger}$ \\ on behalf of the CMS Collaboration \\ Seoul National University, Republic of Korea \\ E-mail: benjamin@cern.ch
}

\begin{abstract}
Many models beyond the standard model, such as the Sequential Standard Model, Grand Unified Theories and Randall-Sundrum model of extra dimensions, predict resonances at the TeV energy scale. These resonances can decay to leptons and photons. Results on single lepton, dilepton and diphoton resonances resonances using $36 \mathrm{fb}^{-1}$ or $41 \mathrm{fb}^{-1}$ of $13 \mathrm{TeV}$ proton-proton collisions produced at the LHC and recorded by the CMS experiment are presented.
\end{abstract}

The 39th International Conference on High Energy Physics (ICHEP2018)

4-11 July, 2018

Seoul, Korea

\footnotetext{
* Speaker.

${ }^{\dagger}$ Benjamin Radburn-Smith is supported in part by the National Research Foundation of Korea (NRF) funded by the Korea government (NRF-2018R1A1A1A05077514 and NRF-2015R1A4A1042542).
} 


\section{Introduction}

Many models beyond the standard model (SM) predict resonances at the TeV energy scale. These include spin-0, spin-1 and spin-2 resonances produced in models such as the Sequential Standard Model (SSM) with SM-like couplings, Grand Unified Theories (GUT) with an E6 gauge group such as the $\psi$ model, and the Randall-Sundrum model of extra dimensions leading to Kaluza-Klein graviton excitations.

The use of leptonic and photonic resonances has been a critical tool in searching for signatures of physics. The $W$ and $Z$ bosons as well as the Higgs boson were all discovered using these signatures. The different decay channels provide different advantages for searches; with excellent energy resolution from electrons at high energies, high muon reconstruction efficiency at high momenta, $\tau$ leptons probing couplings to third generation leptons, and photons sensitive to spin-0 resonances. Only when utilising all these final states can we robustly search for heavy resonances.

All analyses presented here use $36 \mathrm{fb}^{-1}$ of proton-proton collisions recorded by the CMS experiment in 2016, with the exception of one analysis using $41 \mathrm{fb}^{-1}$ of data recorded in 2017 which will be explicitly mentioned below. Many of the analyses also focused on producing model independent limits allowing for further reinterpretations not described here.

\section{Single Lepton Channels}

A search for a highly energetic electron or muon along with missing energy was performed [1]. Events containing one lepton with transverse momentum, $p_{\mathrm{T}}$, greater than 130 (53) GeV for the electron (muon) are selected. The event is vetoed if it contains an extra lepton with $p_{\mathrm{T}}>25 \mathrm{GeV}$. For the electron channel the missing transverse momentum, $p_{\mathrm{T}}^{\text {miss }}$, is required to be greater than $150 \mathrm{GeV}$ to avoid an area of phase space which is not well modelled. The analysis uses a transverse mass quantity, $M_{\mathrm{T}}$, based upon the lepton and missing momentum as the discriminating variable. With no observed excess with respect to the SM, upper limits can be set on the mass of $W^{\prime}$ SSM to be greater than 5.2 TeV as shown on the left in Figure 1.
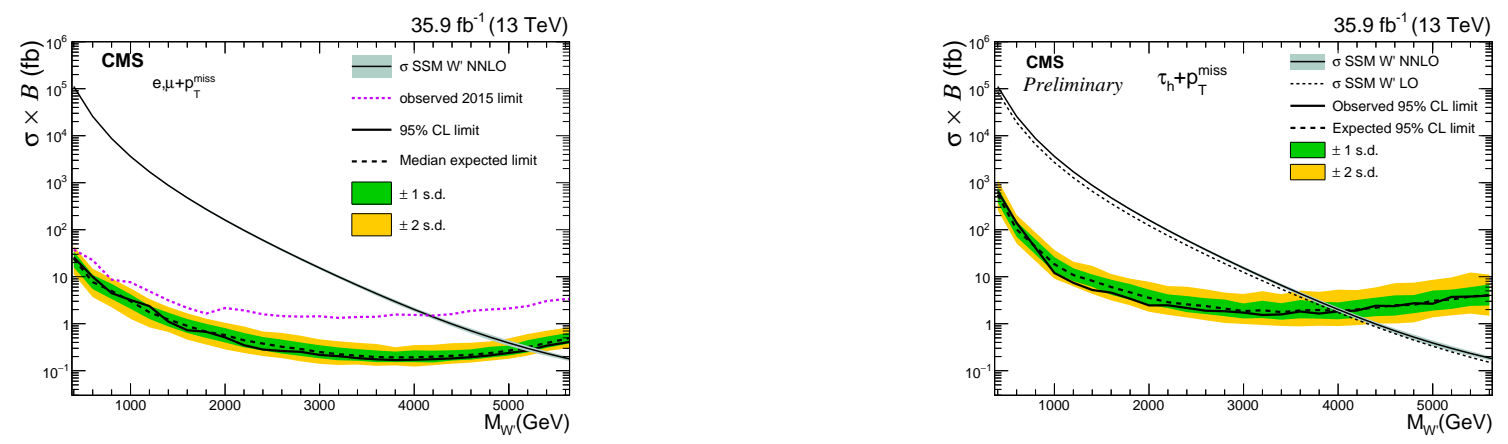

Figure 1: Upper limits at $95 \%$ confidence level for the masses of a $W^{\prime}$ SSM boson decaying to electron or muon plus neutrino (left) and to a $\tau$ plus neutrino (right).

The search for a $W^{\prime}$ boson decaying to a $\tau$ lepton along with missing energy [2] focuses on the hadronically decays of the $\tau$ which result in low charged hadron multiplicity. Leptonically decaying $\tau$ leptons cannot be distinguished from the previous analysis and are covered by that 
analysis. $M_{\mathrm{T}}$ is used as a discriminator variable with the $\tau p_{\mathrm{T}}$ required to be greater than $50 \mathrm{GeV}$ and $p_{\mathrm{T}}^{\text {miss }}$ greater than $90 \mathrm{GeV}$. Events containing additional leptons with $p_{\mathrm{T}}$ greater than $20 \mathrm{GeV}$ are excluded. Figure 1 (right) shows that limits can be set on the mass of $W^{\prime}$ SSM to be greater than 4.0 TeV.

\section{Dilepton Channels}

An inclusive search for a new resonance decaying to pairs of electrons or muons was performed [3]. In this analysis the Monte Carlo (MC) background prediction is normalised to the $Z$ peak and the amount of jet background is estimated from data. Electrons (muons) are required to have $p_{\mathrm{T}}$ greater than $35(53) \mathrm{GeV}$. Improvements to the online and offline muon selection were made with respect to the previous analysis which increased the efficiency for dimuon masses above $1 \mathrm{TeV}$. Limits are set on the mass of the spin-1 resonance $Z_{\text {SSM }}^{\prime}\left(Z^{\prime}{ }_{\psi}\right)$ to be greater than 4.5 (3.9) $\mathrm{TeV}$. Limits are set on the mass of spin-2 resonances with couplings $k / \bar{M}_{\mathrm{Pl}}=0.01,0.05,0.1$ to be greater than 2.1, 3.65, 4.25 TeV, respectively. The limits are shown in Figure 2.
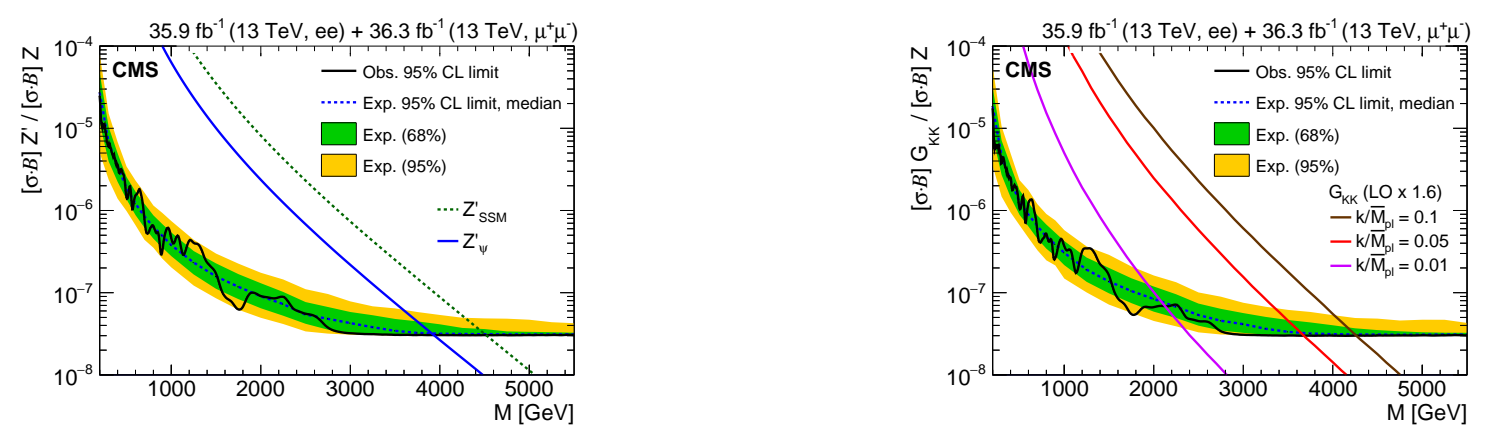

Figure 2: Limits at 95\% confidence level for the masses of spin-1 resonances (left) and spin-2 resonances (right).

An update to the analysis in the electron only channel was performed using $41 \mathrm{fb}^{-1}$ of data recorded in 2017 [4]. The statistical analysis of this search was combined with the previous result to place stronger limits on the spin-1 mass $Z_{\mathrm{SSM}}^{\prime}\left(Z^{\prime}{ }_{\psi}\right)$ to be greater than $4.7(4.1) \mathrm{TeV}$.

A model independent search for heavy resonances decaying to an electron and muon was performed [5]. The lepton thresholds are the same as in the dilepton analysis and the particles are not required to have opposite charge, to avoid efficiency losses through charge misidentification. The dilepton invariant mass is required to be greater than $200 \mathrm{GeV}$. $W+$ jets and QCD multijet backgrounds are calculated from data. The limits are shown in Figure 3 where the mass of a lepton flavour violating (LFV) $Z^{\prime}$ boson, in which the branching fraction to electron-muon decays is set at $10 \%$, should be greater than $4.4 \mathrm{TeV}$.

\section{Diphoton Channels}

A search for resonant production of photon pairs was performed [6]. This is a very clean final state without additional activity in the direction of the photons. The photons are required to have $p_{\mathrm{T}}$ greater than $75 \mathrm{GeV}$ with at least one photon present in the barrel. A fit is performed to the 


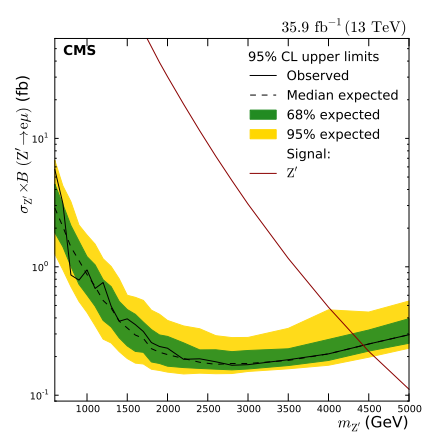

Figure 3: Limits at $95 \%$ confidence level for the masses of a LFV $Z^{\prime}$ boson with branching fraction to electron+muon set to $10 \%$.

invariant mass spectra to determine the compatibility of the data with the background-only and signal+background hypotheses. The results are then interpreted in terms of a spin- 2 resonance and spin-0 heavy Higgs boson resonance. The limits on the mass of spin-2 resonances with couplings $\widetilde{\kappa}=0.01,0.1,0.2$ to be greater than $2.1,4.1,4.6 \mathrm{TeV}$ respectively, as shown in Figure 4 .
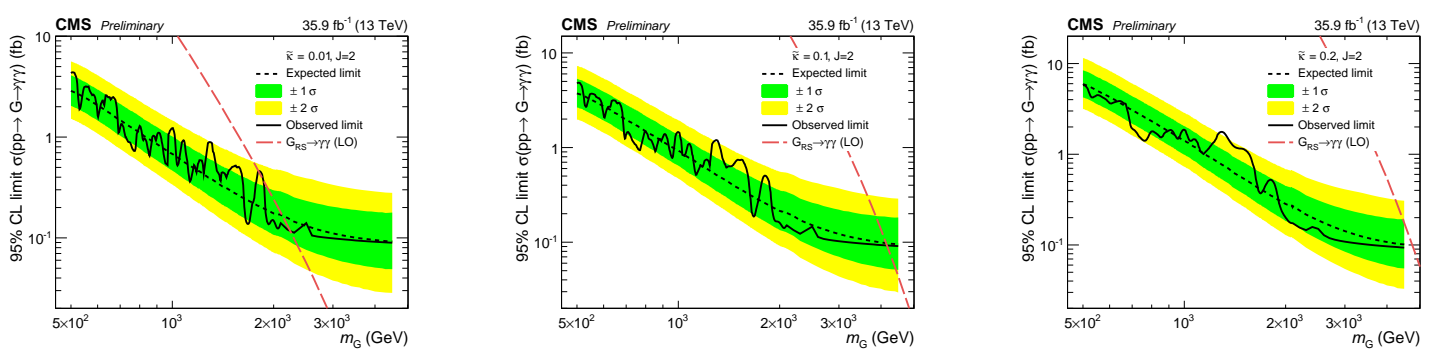

Figure 4: Limits at $95 \%$ confidence level for the masses of a spin-2 resonance with coupling of 0.01 (left), 0.1 (middle) and 0.2 (right).

\section{References}

[1] The CMS Collaboration, "Search for high-mass resonances in final states with a lepton and missing transverse momentum at $\sqrt{s}=13 \mathrm{TeV}$ ", JHEP 06 (2018) 128

[2] The CMS Collaboration, "Search for a $W^{\prime}$ boson decaying to a lepton and a neutrino in proton-proton collisions at $\sqrt{s}=13 \mathrm{TeV}$ ", CMS-PAS-EXO-17-008 (2018), https://cds.cern.ch/record/2621539

[3] The CMS Collaboration, "Search for high-mass resonances in dilepton final states in proton-proton collisions at $\sqrt{s}=13 \mathrm{TeV} "$, JHEP 06 (2018) 120

[4] The CMS Collaboration, "Search for high mass resonances in dielectron final state", CMS-PAS-EXO-18-006 (2018), https://cds.cern.ch/record/2308270

[5] The CMS Collaboration, "Search for lepton-flavor violating decays of heavy resonances and quantum black holes to e $\mu$ final states in proton-proton collisions at at $\sqrt{s}=13 \mathrm{TeV}$ ", JHEP 04 (2018) 073

[6] The CMS Collaboration, "Search for physics beyond the standard model in the high-mass diphoton spectrum at $\sqrt{s}=13 \mathrm{TeV}$ ", CMS-PAS-EXO-17-017 (2018), http://cds.cern.ch/record/2316245 